\title{
A Randomized Controlled Clinical Trial of Dialogical Exposure Therapy versus Cognitive Processing Therapy for Adult Outpatients Suffering from PTSD after Type I Trauma in Adulthood
}

\author{
Willi Butollo ${ }^{a}$ Regina Karl ${ }^{a} \quad J^{\prime}$ ulia König ${ }^{b}$ Rita Rosner ${ }^{b}$ \\ a Department of Psychology, Ludwig Maximilian University, Munich, and ${ }^{\mathrm{b} C l i n i c a l}$ and Biological Psychology, \\ Catholic University of Eichstätt-Ingolstadt, Eichstätt, Germany
}

\begin{abstract}
Key Words
Posttraumatic stress disorder - Gestalt therapy .

Cognitive-behavioural therapy - Treatment outcome .

Randomized trial · Dialogical exposure therapy ·

Cognitive processing therapy
\end{abstract}

\begin{abstract}
Background: Although there are effective treatments for posttraumatic stress disorder (PTSD), there is little research on treatments with non-cognitive-behavioural backgrounds, such as gestalt therapy. We tested an integrative gestalt-derived intervention, dialogical exposure therapy (DET), against an established cognitive-behavioural treatment (cognitive processing therapy, CPT) for possible differential effects in terms of symptomatic outcome and drop-out rates. Methods: We randomized 141 treatment-seeking individuals with a diagnosis of PTSD to receive either DET or CPT. Therapy length in both treatments was flexible with a maximum duration of 24 sessions. Results: Dropout rates were $12.2 \%$ in DET and $14.9 \%$ in CPT. Patients in both conditions achieved significant and large reductions in PTSD symptoms (Impact of Event Scale - Revised; Hedges' $\mathrm{g}=1.14$ for DET and $d=$ 1.57 for (PT) which were largely stable at the 6-month follow-up. At the posttreatment assessment, CPT performed statistically better than DET on symptom and cognition mea-
\end{abstract}

sures. For several outcome measures, younger patients profited better from CPT than older ones, while there was no age effect for DET. Conclusions: Our results indicate that DET merits further research and may be an alternative to established treatments for PTSD. It remains to be seen whether DET confers advantages in areas of functioning beyond PTSD symptoms.

(c) 2015 S. Karger AG, Basel

\section{Introduction}

Current meta-analyses [1-6] as well as practice guidelines [7-9] strongly support trauma-focused methods in the treatment of posttraumatic stress disorder (PTSD), i.e. cognitive-behavioural therapies (CBT) and eye movement desensitization and reprocessing. Even though these efficacious treatments exist, some researchers argue that the development of new treatments for PTSD is still necessary [10], and new treatment approaches emerge, such as writing therapy [11] or Internet-based therapy [12]. New related concepts are being discussed, such as the feeling of being contaminated [13] or moral injury [14], and treatments are being assessed in difficult settings and different cultures [15].

\section{KARGER}

E-Mail karger@karger.com

www.karger.com/pps
(C) 2015 S. Karger AG, Basel

0033-3190/15/0851-0016\$39.50/0 
Research on emotion-focused therapy (EFT) shows that interventions based on gestalt principles can achieve effects comparable to those of CBT interventions $[16,17]$. A review of 6 studies on the efficacy of experiential and gestalt-based treatments for traumatized patients [18] showed that there is a lack of methodologically rigorous research on gestalt-based therapies: the studies reviewed lacked either a control condition, a randomized design or failed to establish PTSD diagnoses.

Even though established treatments are effective, most studies find that a substantial minority of patients fail to profit from them and a substantial minority drop out. This makes it worthwhile to explore other treatment options that may be either more acceptable to patients in general, or offer an alternative for those not able to engage in or profit from CBT. A current meta-analysis of dropout from treatment for PTSD found an average dropout rate of $18 \%$ from active treatments, with considerable variability across studies [19].

\section{Dialogical Exposure Therapy for PTSD}

Dialogical exposure therapy (DET) is based on gestalt therapy and therefore relies strongly on gestalt theory which understands the self as a dynamic cognitive-emotional state that constantly constitutes itself as a result of interactional experience with oneself and the environment. Therefore the term 'self-processes' $[20,21]$ is preferred. Usually, a person is aware of the situation as well as of her- or himself (self-awareness), has knowledge about her- or himself (self-representation), can tolerate conflicts with the environment, and is able to devise interactions according to needs, opportunities and previously acquired interaction skills (contact ability). A traumatic experience, that is an incident or interaction that is perceived as life-threatening, can heavily distort these processes. In such situations, survival may be only possible by submitting to the premises of a perpetrator (surrender reflex, confluence) by giving up one's sense of self ('self-collapse'). This does not only hold for interpersonal trauma, but also for threat experienced as coming from outer sources, including natural catastrophes. The selfcollapse is rewarded by a reduction of the threat resulting from being different from the aggressor or having different values, but survival has the cost of losing one's sense of self. In the following period, the event is cognitively repeated over and over in an attempt to resolve it, and many trauma survivors succeed in integrating their experience into their self-processes. In PTSD however, the

Dialogical Exposure Therapy versus

Cognitive Processing Therapy for PTSD cognitive rehearsals only lead to repetitions of the selfcollapse as the only available tool to reduce the seemingly intolerable fear. The process generalizes such that all arousal experiences, not only the ones triggered by direct trauma reminders, lead to the same submissive coping attempt by self-collapse. As a consequence, self-representation deteriorates, leading to social withdrawal, feelings of insecurity and a chaotic, dissolved self as contact between the different self-processes as well as between the person and the environment deteriorates. The self is then unable to shape its interactions in a satisfying way, especially when under stress. This is true for stress stemming from any source: for a traumatized patient, everyday conflicts which have been easily resolved before can be highly problematic.

The overall goal of DET is to identify and resolve the disruptions of contact so that the patient will be able to experience continuity in his or her self and regain the ability to shape interactions with the environment (for a more in-depth discussion of DET principles, see [22]).

DET has been developed at a university-based outpatient clinic since the 1990s. It has similarities to EFT, as both share a background in gestalt theory, but DET was developed for patients meeting criteria for PTSD, while EFT focuses more broadly on emotional trauma. DET includes exposure to the traumatic event while in EFT, patients are encouraged to focus on and resolve difficult emotions. Therapists are more active and directive than EFT therapists. In contrast to cognitive-behavioural therapies (CBTs), however, DET therapists are directive in supporting their patients in exploring their symptoms phenomenologically and in accepting themselves unconditionally with their symptoms and deficits. This self-acceptance is the condition for and precursor of symptomatic change. The therapy is structured into four phases: safety, stability, confrontation and integration. The phases overlap and can have different emphases depending on the type of trauma. Their timing depends on the individual patients' processes. Phase I is about perception of the outside world and differentiation of the perception of safety as well as the acceptance of feelings of uncertainty. Phase II focuses on inner perception (self-awareness of the feeling/thought interactions) and stabilization in everyday conflict episodes. Phase III contains the exposure to the trauma. In addition to discussing the event and visiting the place where it happened, if feasible, this phase includes the core intervention of DET, dialogical exposure. While the first two steps are similar to CBTs, dialogical exposure is not about habituation, but about finding a way of preserving 
one's self-processes while in contact with the perpetrator. In a modified version of empty chair work, the patient is encouraged to enter into an interactional confrontation with the imagined presence of the experience, for example with the perpetrator(s), the suicide or the (natural) disaster. The focus here is on the patient's taking responsibility for him- or herself, and this can also mean drawing back from the encounter (another difference to CBT). In phase IV, the focus is on accepting the experience and the changes resulting from it, as well as on relapse prevention. The generalization of skills and gains from the therapy setting into everyday life are further goals.

DET has so far been tested in two studies. One involved Bosnian women who had suffered traumatic bereavement and were treated in a group format [23]. Effect sizes of the DET intervention were: Cohen's $d=0.66$ for PTSD symptoms and $d=0.50$ for traumatic grief in the intent-to-treat (ITT) sample. While these effects seem modest, and were indeed only slightly better than the control group given supportive therapy, the effect on PTSD symptoms is well within the range of the overall uncontrolled effect size of group treatment for PTSD as computed in a recent meta-analysis [24] $(d=0.71,95 \%$ CI $0.51-0.91)$. Also, this was a sample with a high level of chronicity and still living in postwar conditions.

Before attempting this large study, we conducted a pilot study of DET in an individual therapy setting with 25 treatment-seeking patients suffering from PTSD after adulthood trauma who received thirteen 90 -min sessions of DET [25]. Pre-post effect sizes were large for PTSD symptoms $(d=0.95$ for the Impact of Event Scale - Revised, IES-R [26] and $d=0.70$ for the Posttraumatic Diagnostic Scale, PDS [27]) and moderate for general psychopathology and interpersonal problems. The therapy was accepted well by the patients (dropout rate 16\%). Therefore we decided to test DET in a larger sample. For ethical reasons, we decided not to include an untreated control group but rather an active control group. For this, we chose cognitive processing therapy (CPT) [28] for several reasons: the efficacy of CPT is well established for PTSD after different types of traumas. It is manualized, which makes it easy to teach and learn, and we had the opportunity of attending a workshop taught by the author of CPT, Patricia A. Resick, who authorized the translation of the manual and supported the implementation.

Our first hypothesis was that both therapies would be efficacious in reducing PTSD symptoms (primary outcome), general psychopathology and negative trauma- related cognitions (secondary outcome). Secondly, we expected that life satisfaction would improve and interpersonal problems decrease in both therapies (tertiary outcomes). Thirdly, we aimed to compare treatments to explore whether they performed differently on any of these measures. As this is the first comparison study using DET, we did not have a hypothesis concerning PTSD symptoms and general distress, but we expected CPT to effect more change in posttraumatic cognition and DET in interpersonal distress and life satisfaction.

\section{Method}

\section{Participants}

Participants were recruited from consecutive patients seeking treatment at a university-based outpatient clinic between September 2008 and July 2011. Our enrolment goal was 90 participants total, which would have given us a power of 0.65 to detect a difference with a medium effect size between therapies (estimated with G*Power 3 [29]). However, we were able to include more patients because the number of eligible patients was higher than expected. If a trauma history was present as assessed in an initial telephone screening, patients were invited for an intake interview, in which one of two experienced master level clinicians assessed them for eligibility. Patients were enrolled in the study if they had a primary diagnosis of PTSD according to the Diagnostic and Statistical Manual of Mental disorders, 4th edition (DSM-IV) [30] due to a traumatic event that had happened at least 1 month previously. Patients were considered not to have a primary diagnosis of PTSD if their symptoms were overshadowed by dissociative identity disorder or severe personality disorder. They were excluded if they reported ongoing distress from psychosis or substance dependence or if their symptoms were due to childhood abuse or neglect. We excluded such patients because we considered them as probably needing more intense and/or longer treatment than was offered in the study. We followed the ethics committee's recommendation to exclude highly comorbid patients as there is limited prior experience with gestalt therapy for such patients in the literature. With respect to depression, we excluded patients for whom depression predated the trauma and for whom in the clinical assessment prior to study intake the clinician had rated the patient to be unable or not to wish to commit to treatment within the stated study protocol. Additional exclusion criteria were organic brain syndrome, current psychotherapy, lack of German language skills (as determined in the intake interview), current suicidality and being younger than 18 years. At the end of this interview, patients received baseline self-report measures for completion at home. In two subsequent diagnostic sessions, another master level clinician collected these self-report measures and assessed for comorbid axis I disorders.

Figure 1, the CONSORT chart, shows the flow of patients through the study. Of 181 prospective patients invited to the initial interview, 33 met exclusion criteria or did not meet inclusion criteria and were offered treatment outside the study protocol, leaving 148 patients who were enrolled. In the CPT condition, each therapist's first case was considered a training case and was not included in the analyses. As these pilot cases were not taken into
18

Psychother Psychosom 2016;85:16-26 DOI: $10.1159 / 000440726$
Butollo/Karl/König/Rosner 


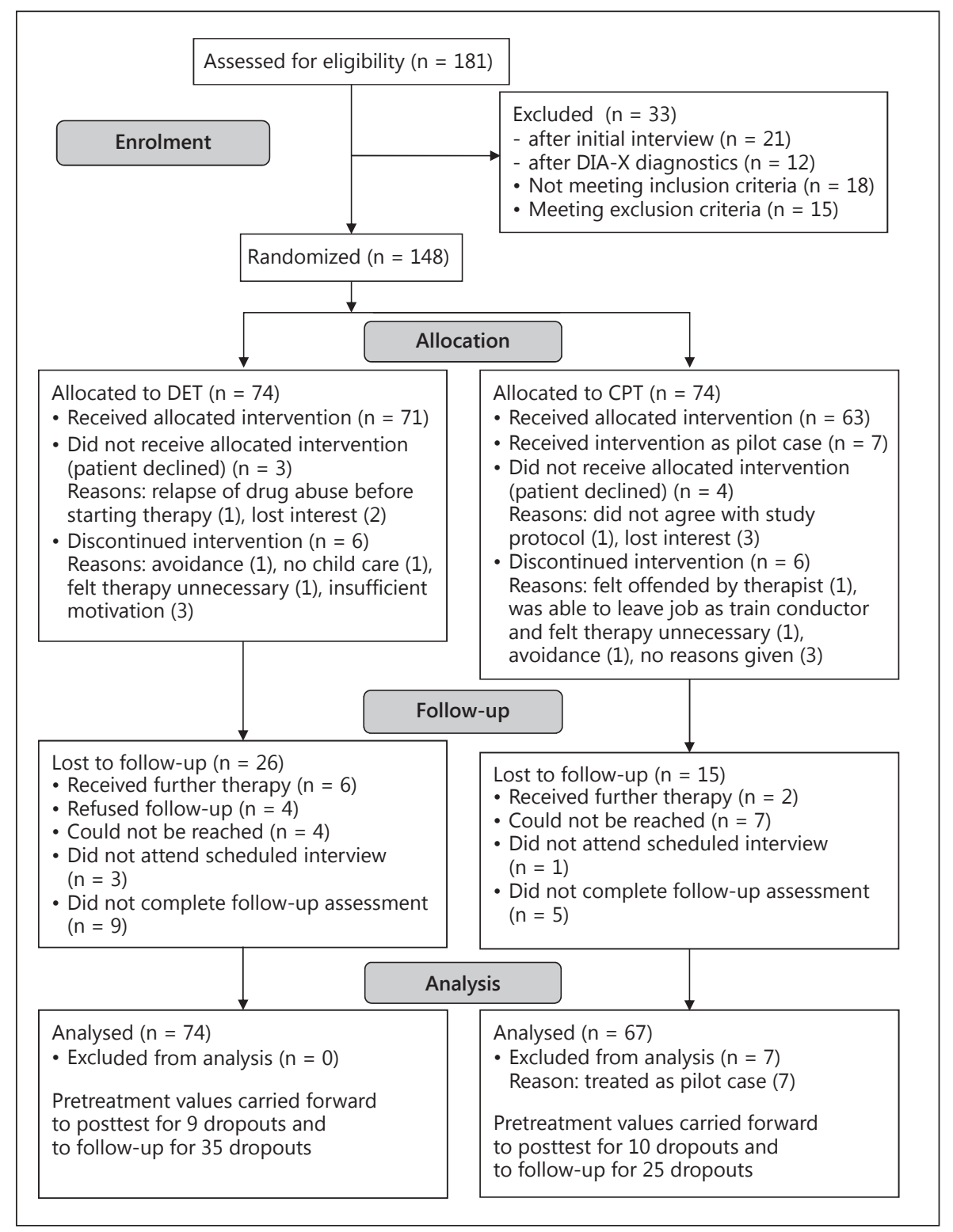

Fig. 1. CONSORT flow chart.

account when generating the randomization sequence, the DET ITT sample was larger than the CPT sample.

The ITT sample therefore consisted of 141 patients, 74 in DET, and 67 in CPT. Sample characteristics are given in table 1 (more detailed information to be found in the online suppl. table 1; see www.karger.com/doi/10.1159/000440726, for all online suppl. material). Randomization was largely successful, with only few significant differences between the two subsamples. DET patients were significantly older than CPT patients, with $\mathrm{t}(139)=2.271$, $p=0.025$. Therefore, age was included in analyses as a covariate. CPT patients endorsed significantly more symptoms of obsessivecompulsive personality disorder $[\mathrm{DET}$, mean $=3.73, \mathrm{SD}=1.76$; $\mathrm{CPT}$, mean $=4.40, \mathrm{SD}=1.69 ; \mathrm{t}(133)=-2.255, \mathrm{p}=0.026]$, but as this variable showed no connection to symptomatic improvement, it was not included in the analyses.

Dialogical Exposure Therapy versus Cognitive Processing Therapy for PTSD

\section{Measures}

At study entry, PTSD was assessed with the German version of the International Diagnostic Checklists for DSM-IV and ICD (IDCL) [31], and comorbid DSM-IV axis I diagnoses were established using the DIA-X [32], a German computer-assisted and modified version of the Composite International Diagnostic Interview with an excellent reliability (93.3\% agreement, $\kappa=0.76)$ [33]. The IDCL have also been demonstrated to be reliable, with agreements between $\kappa=0.67$ and $\kappa=0.88$ for different interviewers [34]. We screened for axis II disorders using the SCID-II questionnaire [35]. If a patient scored above the cut-off for a personality disorder, we used clinical judgement to determine whether exclusion criteria were met.

The IES-R [36], a self-report instrument that measures the intensity of PTSD symptoms, was our primary outcome measure. It was administered by the therapist before each session as a process 
Table 1. Characteristics of the two treatment groups

\begin{tabular}{lll}
\hline Variable & $\begin{array}{l}\text { DET } \\
(\mathrm{n}=74)\end{array}$ & $\begin{array}{l}\text { CPT } \\
(\mathrm{n}=67)\end{array}$ \\
\hline Age range, years & $18-78$ & $19-53$ \\
\hline Age, years ${ }^{\mathrm{a}}$ & $37.99 \pm 12.1$ & $33.67 \pm 10.3$ \\
Number of traumatic events & $3.1 \pm 1.9$ & $2.7 \pm 1.6$ \\
Months since index trauma & $18.65 \pm 25.38$ & $19.08 \pm 25.22$ \\
\hline Female & $48(64.9)$ & $45(67.2)$ \\
Current comorbid diagnosis & $31(41.9)$ & $35(52.2)$ \\
$\quad$ Affective disorder & $32(43.2)$ & $33(49.3)$ \\
Anxiety disorder & $10(13.5)$ & $10(14.9)$ \\
Somatoform disorder & $6(8.1)$ & $9(13.4)$ \\
Other & $21(28.4)$ & $15(22.4)$ \\
$\quad$ None & & \\
Trauma type (index trauma) & $19(25.7)$ & $22(32.8)$ \\
Interpersonal & $26(35.1)$ & $28(41.8)$ \\
Accident & $7(9.5)$ & $5(7.5)$ \\
Loss & $4(5.4)$ & $1(1.5)$ \\
Medical issue & $18(24.3)$ & $11(16.4)$ \\
Other & & \\
\hline
\end{tabular}

Results are expressed as means \pm SD or numbers with percentages in parentheses.

${ }^{a}$ This difference was statistically significant, with $t=2.271$, d.f. $=139$ and $\mathrm{p}=0.025$.

measure, as well as before and after treatment and at the follow-up. The psychometric properties of the German version of the IES-R are good and comparable to the original version [26].

As a second primary outcome measure, we used the PDS [27], a self-report instrument which can be used for determining PTSD diagnostic status according to DSM-IV criteria as well as symptom severity. Its psychometric properties are good, although the German version has a tendency to overdiagnose [37]. For diagnosis scoring, we used a combination of symptom cluster scoring according to DSM-IV criteria and a severity cutoff of 18 [38].

As we were interested not only in PTSD symptoms, but also overall psychological functioning, we used the Brief Symptom Inventory (BSI) [39] as a secondary outcome measure. The BSI is a self-report measure for a broad range of psychological problems. The German version [40] has good psychometric properties [41]. We used the Global Severity Index before and after therapy and at the follow-up to assess change of overall psychological distress.

The Posttraumatic Cognitions Inventory (PTCI) [42] is a 33item self-report measure for the assessment of trauma-related cognitions. It was administered before and after treatment and after 6 months, as well as after every fourth session. As the PTCI highly correlates with PTSD symptoms, but is not a PTSD measure, we considered it a secondary outcome.

The design included two exploratory outcomes. The Inventory of Interpersonal Problems - Circumplex Version [43] (IIP-C) is a measure of interpersonal problems. It is sensitive to change and has been used in psychotherapy research both as an outcome measure and as a predictor of outcome [44].
In order to assess life satisfaction, we used a shortened version of an existing questionnaire [45], which was in turn adapted from an older measure [46]. Our measure consisted of 19 items measuring satisfaction in the areas social situation, health, own person, leisure time and financial status. Psychometric data on the specific items used in this study are not available. However, Cronbach's a for our sample ( $\mathrm{n}=115$, reflecting the exclusion of cases with missing data) was, at 0.90 , very good. Information on further process measures and tertiary outcomes has been documented in the study protocol, which can be obtained from the first author.

The follow-up about 6 months after the end of therapy consisted of a battery of self-report instruments that were mailed to the patients. They were asked to return the completed instruments during an interview conducted by a staff member.

\section{Treatments}

The principles and structure of DET have already been described above. The therapy was carried out according to the manual [47]. CPT [28] is one of the best-established CBTs for PTSD. It is a highly structured, predominantly cognitive therapy protocol targeting dysfunctional cognitions about the traumatic event, oneself and others. In this study we used a translated and slightly modified manual [48]. In addition to the written exposure component, we included behavioural homework to help patients counteract behavioural avoidance. Such homework does not disagree with the rationale behind CPT and has been added before [49].

Treatment length was flexible with a maximum duration of 24 sessions. If a patient had completed the therapy ( 15 sessions in the CPT condition or the four phases in the DET) and patient, therapist and supervisor agreed that sufficient improvement had been achieved, treatment was terminated. This increases external validity as in real life therapies are usually not continued until a fixed session number is reached. Only a minority of cases terminated therapy early. In both conditions, patients were offered up to 2 'booster sessions' which were optional and could be scheduled at any time during the 6-month follow-up period. We decided to offer this because it has been our experience that it can be very helpful for patients to know that they can get support during difficult times they anticipate, such as anniversaries of traumatic events or court dates.

\section{Therapists}

Eight master level psychologists conducted therapies in the DET condition, 2 of them male. Their ages ranged from 28 to 55 years, their clinical experience at the beginning of the study from 1 to 15 years. Two had completed gestalt therapy training in an external institution, and 2 completed this training during the course of the study. Five were in training to become licensed CBT therapists ( 3 of them completing during the time of the study). All DET therapists had completed a 96-hour curriculum on treating traumatized patients, of which DET was the main focus.

Seven female therapists treated patients in the CPT condition. Their ages ranged from 27 to 45 years and their clinical experience from 1 to 10 years at study entry. The CPT therapists were all master level psychologists in training to become licensed CBT therapists (4 finished during the study). Three completed a 2-day CPT workshop and a supervision session with Patricia A. Resick; the other 4 therapists joined the study later and were trained individually by the third author who had attended an additional 2 days of CPT workshops with Patricia A. Resick and translated the CPT 
Table 2. Scores at the three measurement points in the two treatment conditions (ITT sample) using LOCF

\begin{tabular}{|c|c|c|c|c|c|c|c|c|c|c|c|c|}
\hline \multirow[t]{2}{*}{ Measure } & \multicolumn{6}{|l|}{$\operatorname{DET}(\mathrm{n}=74)$} & \multicolumn{6}{|l|}{$\mathrm{CPT}(\mathrm{n}=67)$} \\
\hline & mean & $\mathrm{n}$ & mean & $\mathrm{n}$ & mean & $\mathrm{n}$ & mean & $\mathrm{n}$ & mean & $\mathrm{n}$ & mean & $\mathrm{n}$ \\
\hline IES-R & $69.22(16.07)$ & 70 & $40.06(32.31)$ & 72 & $36.47(30.69)$ & 72 & $66.76(19.36)$ & 63 & $27.96(28.98)$ & 66 & $29.91(28.77)$ & 66 \\
\hline PDS & $30.08(9.89)$ & 71 & $18.79(14.02)$ & 72 & $16.88(13.83)$ & 72 & $28.56(10.15)$ & 66 & $15.93(12.36)$ & 67 & $16.03(13.17)$ & 67 \\
\hline BSI GSI & $1.48(0.68)$ & 70 & $0.97(0.89)$ & 71 & $0.88(0.82)$ & 72 & $1.45(0.74)$ & 66 & $0.81(0.71)$ & 67 & $0.82(0.75)$ & 67 \\
\hline IIP & $1.34(0.59)$ & 66 & $1.09(0.66)$ & 69 & $0.98(0.63)$ & 70 & $1.38(0.57)$ & 66 & $1.13(0.64)$ & 67 & $1.11(0.60)$ & 67 \\
\hline
\end{tabular}

Results are expressed as means with SD in parentheses. LOCF = Last observation carried forward; GSI = Global Severity Index; LS = life satisfaction.

manual. Training included reviewing the treatment manual together session by session, and attending supervision a few times before taking cases. To ensure that therapists in the CPT condition were no less qualified in conducting trauma treatment in line with the treatment manual, the first case of each CPT therapist was considered a pilot case. This case was not included in analyses and received especially close supervision. It is unusual to draw training cases from a randomized study sample, but we wanted the training cases to be relevant to the study population, and this procedure enabled us to start study treatments in the DET condition (also, some CPT therapists joined the study at a later date).

Therapists in both conditions were supervised during the study on a regular basis (every fourth session on average). Supervision included discussion of therapist adherence and patient progress. Every case was discussed, not only problematic ones. Between supervisions therapists met weekly for case discussions. DET therapists were supervised by the first author, CPT therapists by the fourth author. For the first 115 cases (80\%), all sessions were videotaped. Later cases were videotaped less frequently and mainly for use in supervision. In the DET condition, the first 2 authors reviewed at least 1 case per therapist with the help of the video tapes in order to ensure adherence to DET principles. This was felt to be necessary only in the DET condition, because most therapists also had training in CBT, while none of the CPT therapists had any training in DET. It is difficult to compare the amount of training between therapist groups. In contrast to DET, there was no longstanding tradition of conducting CPT at the institution, and as the manual was translated specifically for this study, therapists could not have long-standing experience with it. However, the theoretical principles and treatment components of CBT but not of DET are routinely taught in psychology university programmes, and all CPT therapists were in CBT training, so they were considered to be ready to treat study patients after the training described above and the pilot case.

To ensure adherence, therapists in both conditions were required to complete a detailed documentation of the interventions used in each session. The study procedure was approved by the institution's ethics committee.

\section{Data Analysis}

We analysed the effects of treatment on primary, secondary and tertiary outcomes using separate 2 (intervention) $\cdot 3$ (time point) repeated measures MANOVAs with participant age as a covariate.
Missing data were replaced with the last available score (last observation carried forward). This is a conservative procedure because it assumes no change has happened in patients with missing data at posttreatment and/or follow-up examinations. For significant effects in the MANOVAs, we conducted appropriate post hoc tests (ANOVAs and t tests). We calculated Hedges' g effect sizes from pre- to posttreatment and pretreatment to follow-up time points. In order to compare treatments, we calculated between-group effect sizes as described by Klauer [50] using an online calculator [51].

\section{Results}

At the pretreatment assessment, the two subsamples were similar in terms of clinical severity. The sample exhibited marked psychopathology. The mean BSI Global Severity Index corresponded to a t value of above 80 according to norms [40] and was significantly higher than the mean of a sample of German patients seeking outpatient psychotherapy [41] [mean $=1.14, \mathrm{SD}=0.67$, $\mathrm{t}(135)=5.321, \mathrm{p}<0.001]$. The mean PDS severity, at 29.35 ( $\mathrm{SD}=10.0)$, was in the moderate to severe range. Means for the three IES-R symptom clusters were in the severe range (above 19). Interpersonal distress, on the other hand, was rather low and did not differ significantly from the healthy German norm: mean $=1.28, \mathrm{SD}=$ $0.52, \mathrm{t}(131)=1.504, \mathrm{p}=0.135$ [52]. Mean scores and standard deviations for both treatment groups (ITT sample) are given in table 2 .

Drop-out rates at the posttreatment assessment were $12.2 \%$ for DET (4.1\% of those allocated to DET did not start treatment, $8.1 \%$ dropped out of treatment) and $14.9 \%$ for CPT (6.0\% declined treatment after allocation, $9.0 \%$ dropped out of treatment). The difference was not statistically significant, with $\chi^{2}(1)=0.230, p=0.631$. At the 6-month follow-up, study drop-out rates were markedly higher, increasing the overall study dropout to $47.3 \%$ 
Table 3. Repeated measures ANOVAs for primary outcomes (ITT sample)

\begin{tabular}{llrlrr}
\hline Measure & Effect & MS & d.f. & F & p \\
\hline IES-R & time & $14,607.679$ & 1.546 & 42.075 & $<0.001^{\mathrm{a}}$ \\
& age & $9,576.554$ & 1 & 6.599 & $0.011^{\mathrm{a}}$ \\
& intervention & $14,401.526$ & 1 & 21.855 & $<0.001^{\mathrm{a}}$ \\
& time $\times$ intervention & $2,763.634$ & 1.546 & 7.960 & $0.001^{\mathrm{a}}$ \\
& time $\times$ age & $2,559.062$ & 1.546 & 7.371 & $0.002^{\mathrm{a}}$ \\
& age $\times$ intervention & $15,977.865$ & 1 & 11.010 & $0.001^{\mathrm{a}}$ \\
& time $\times$ age $\times$ intervention & $2,321.073$ & 1.546 & 6.685 & $0.004^{\mathrm{a}}$ \\
\hline PDS & $1,315.480$ & 1.395 & 16.842 & $<0.001^{\mathrm{a}}$ \\
& time & 705.753 & 1 & 2.049 & $0.155^{\mathrm{a}}$ \\
& age & 871.998 & 1 & 2.531 & $0.114^{\mathrm{a}}$ \\
& intervention & 304.219 & 1.395 & 3.895 & $0.036^{\mathrm{a}}$ \\
time $\times$ intervention & 105.163 & 1.395 & 1.346 & $0.258^{\mathrm{a}}$ \\
time $\times$ age & 778.130 & 1 & 2.259 & $0.135^{\mathrm{a}}$ \\
& age $\times$ intervention & 283.711 & 1.395 & 3.632 & $0.028^{\mathrm{a}}$ \\
time $\times$ age $\times$ intervention & & & &
\end{tabular}

MS = Mean squares; d.f. $=$ degrees of freedom, adjusted according to Greenhouse-Geisser.

${ }^{a}$ For these effects, the MANOVA including both measures was significant.

Table 4. Effect sizes (Hedges' g) in the ITT sample (last observation carried forward)

\begin{tabular}{|c|c|c|c|c|c|c|}
\hline \multirow[t]{2}{*}{ Measure } & \multicolumn{3}{|c|}{$\begin{array}{l}\text { Pretreatment vs. } \\
\text { posttreatment values }\end{array}$} & \multicolumn{3}{|c|}{$\begin{array}{l}\text { Pretreatment vs. } \\
\text { follow-up values }\end{array}$} \\
\hline & DET & CPT & between $^{a}$ & DET & $\mathrm{CPT}$ & between $^{a}$ \\
\hline IES-R & 1.14 & 1.57 & 0.25 & 1.33 & 1.50 & 0.08 \\
\hline PDS & 0.93 & 1.12 & 0.06 & 1.10 & 1.07 & -0.09 \\
\hline BSI & 0.64 & 0.88 & 0.16 & 0.80 & 0.85 & 0.03 \\
\hline PTCI & 0.65 & 1.03 & 0.26 & 0.79 & 0.98 & 0.12 \\
\hline LS & 0.22 & 0.29 & 0.05 & 0.30 & 0.37 & 0.04 \\
\hline IIP & 0.40 & 0.41 & 0.01 & 0.59 & 0.46 & -0.14 \\
\hline
\end{tabular}

LS = Life satisfaction.

${ }^{\text {a }}$ Calculated as $\mathrm{d}_{\text {korr }}$ according to Klauer [50]. Effect sizes with negative indices are in favour of DET, with positive indices in favour of CPT.

in the DET and $37.3 \%$ in the CPT condition. It is important to note that these dropouts occurred after the therapies had ended; therefore their clinical meaning is limited, but the high attrition makes interpretation of differences between therapies at follow-up difficult.

Although we conducted three MANOVAS for the different types of outcomes, ANOVA outcomes are given in table 3 because they are more easily interpreted. For reasons of space, only the primary results table is included here, while secondary and tertiary outcomes can be accessed online (online suppl. table 2a, b). Each effect that was significant in the original MANOVA is indicated. For all 6 outcome measures, there was a main effect of time, and post hoc $t$ tests showed, as could be expected, that scores were significantly worse at pretreatment than at either posttreatment or follow-up examinations, while the latter two did not differ significantly. Pre-post effect sizes are given in table 4 . Effects in both conditions were large for PTSD measures and general psychopathology, and moderate to large for posttraumatic cognitions [53].

There was a main effect for age with an advantage for younger patients on IES-R, BSI Global Severity Index and PTCI. There was also a main effect for intervention on the IES-R and BSI, indicating higher scores in the DET sample. More interestingly, there was a significant interaction effect of time and intervention (indicating that patients in the two treatments changed differently) for IES-R, PDS, BSI and PTCI. Post hoct tests comparing the two intervention groups at the three time points were all non-significant, however, except for posttreatment IES-R, where CPT patients scored significantly lower, with $\mathrm{t}(131)=2.309, \mathrm{p}=$ 0.022 . As can be seen in table 2 , all scores in all four measures decreased between pre- and posttreatment assessments for CPT and slightly increased to follow-up, while in DET, they showed a further decline, which probably explains the significant effects. Looking at the effect sizes in table 4, it becomes apparent that even though betweentreatment effect sizes were rather small, they were all in favour of CPT after treatment. At the follow-up, this difference had become much less noticeable with most between-treatment effect sizes below 0.01 . The two exceptions to this are the PTCI, where there is a small advantage for CPT, and the IIP where DET patients did slightly better.

There was a significant interaction effect of time and age for the IES-R such that younger patients improved more. Even though for a few other measures this interaction was significant, the original MANOVAS were not. Interestingly, the three-way interaction effect of age, time and intervention was significant for all three MANOVAs and for all post hoc ANOVAs except life satisfaction. In order to shed light on the three-way interaction, we conducted the same MANOVAs separately for the two intervention groups, again with time as a covariate. For DET, only the main effect of time was significant for IES-R and PDS. No other main or interaction effect reached significance. For CPT, there was a significant main effect of time on all measures, as well as a main effect of age on IES-R, PTCI and life satisfaction (in all cases younger patients had the advantage). There was a significant interaction 
effect of time and age for IES-R, BSI, PTCI and IIP. For these measures, we split the sample into older and younger patients using the median and compared scores at the three time points. On IES-R, BSI and PTCI, there were no differences between age groups at the pretreatment examination, while younger patients had significantly lower scores at the follow-up. On IIP, there were no significant differences between younger and older patients at any time point. Therefore, on most measures, younger CPT patients showed more improvement than older ones, while there was no such difference in DET.

Possibly one reason for the significant differences in change over time is that among patients who had completed therapy, significantly more posttests were missing in DET (13) than in CPT (1), with $\chi^{2}(1)=9.952, p=0.002$. Even though the difference was not significant at the follow-up [DET: 26, CPT: $15, \chi^{2}(1)=2.549, \mathrm{p}=0.110$ ], this makes the interpretation of results difficult. Therefore, we ran additional ANOVAs for each outcome, including only patients with complete data from all assessments. This reduced sample size considerably (to 30-32 in DET, $36-41$ in CPT) and resulted in no time therapy interaction effects for any measure.

As there was no clinical rating of PTSD diagnosis at posttreatment or follow-up assessments, we used the PDS for this purpose. Using the last observation carried forward, remission rates were $52.7 \%$ in DET and $61.2 \%$ in CPT, which slightly improved to 59.5 and $64.2 \%$, respectively, at the follow-up. Differences between therapies were not significant [posttreatment time point: $\chi^{2}(1)=$ $1.033, \mathrm{p}=0.309$, follow-up: $\left.\chi^{2}(1)=0.331, \mathrm{p}=0.565\right]$.

We calculated the reliable change index [54] to divide the sample into three groups: reliably improved, no reliable change and reliable deterioration. Detailed results can be accessed online (online suppl. table 3 ). We conducted a $2 \times 2 \chi^{2}$ test for each measure comparing successful (reliably improved) with not successful (no change or deterioration) patients. For IES-R, there was a significant difference in favour of CPT $\left(\chi^{2}=6.145, p=0.013\right)$ but not for PDS $\left(\chi^{2}=0.215, \mathrm{p}=0.643\right)$ or BSI $\left(\chi^{2}=0.445\right.$, $\mathrm{p}=0.505)$.

\section{Discussion}

To our knowledge, this is the first PTSD treatment study comparing a gestalt-based integrative treatment with a CBT. Results show that both therapies had large effects on self-rated PTSD symptoms and general psychological functioning. Effect sizes for IES-R were larger than those

Dialogical Exposure Therapy versus

Cognitive Processing Therapy for PTSD found for psychotherapy in general in a recent meta-analysis [6] of 1.14 (95\% CI 0.97-1.3), while PDS effect sizes fell within this range for CPT and slightly below for DET. When compared to the meta-analytic results for CPT specifically $(1.69,95 \%$ CI 1.27-2.11), the IES-R result for the CPT group corresponded very well, while the PDS effect size fell short. Even though we found significant time-therapy interactions for the primary and secondary outcomes, the only significant difference between the two groups at any time point was posttreatment IES-R, where CPT patients had lower symptom scores. This may be due to more missing data at posttreatment assessments in the DET group. At the follow-up, between-group effect sizes were generally very small. The largest were for posttraumatic cognitions (in favour of CPT) and interpersonal functioning (in favour of DET). These differences are in accordance with the proposed mechanisms of change of the two therapies: DET focuses on the therapeutic relationship, but also other relationships in the patients' lives, while CPT has a strong cognitive focus. However, as the differences between therapies were small and the sample was in the nonclinical range before treatment, this possible long-term advantage of DET may not generalize to patients with clinically significant interpersonal disturbance. Also, a different set of analyses did not indicate differential patterns of response for the two treatments [55]. As the high amount of missing data especially in the DET group at the posttreatment assessment made relying solely on ITT data seem unfair, we also conducted analyses with available data only. This yielded no significant differences between therapies; however, it is possible that this is a disadvantage to CPT, because less successful patients may have been less likely to participate in posttreatment assessments.

Even though we included age as a covariate originally only to control for a pretreatment difference between treatment groups, we found that younger patients profited better than older patients from CPT while there was no such effect for DET. This ties in with earlier results: in a study comparing CPT to prolonged exposure, younger patients profited more from CPT than older ones while the reverse was true for prolonged exposure [56]. In a different set of analyses with our sample, we also found that the efficacy of DET was less dependent on patient characteristics: while pre-post differences in CPT were correlated with patient interpersonal dominance, this was not true for DET [57]. It is possible that the reliance of CPT on written work makes it easier for younger people to engage.

Drop-out rates were low in both therapies (12.2\% in DET and $14.9 \%$ in CPT) and did not differ between the 
two groups, indicating that both interventions were well tolerated by patients. These rates are slightly lower than those found for active PTSD treatments [19]. There are several possible reasons for this, such as the flexible dose of therapy, the low interpersonal psychopathology and comorbid pathology (due to exclusion criteria, especially the exclusion of patients with childhood trauma) in our sample and the fact that therapists had more time than in typical studies to focus on issues of motivation and commitment.

An important strength of the study is its high ecological validity: It took place in a 'real-world' setting, that is the therapies were financed by the patients' health care insurance and there was no grant money. Patients were recruited among treatment seekers at the clinic. Most therapists were still in training for their licenses and clinical experience was mixed. Also, the duration of the study therapies corresponded to the typical length of shortterm treatments in Germany (short-term CBT is 25 sessions, and we saved one as a booster session) and we used a flexible treatment dose. Pretreatment PTSD scores were comparable to those found in other studies [11, 58, 59]. However, with the exclusion of patients with childhood trauma, we did have a sample with less interpersonal pathology than other PTSD studies; in fact, patients did not report raised levels of interpersonal distress.

The study has several limitations. The most important methodological one is our sole reliance of self-report of PTSD symptoms at posttreatment and follow-up time points, especially because the German version of the PDS tends to overdiagnose [37]. Thus, our results with respect to remission rates may not be reliable.

Some participants were only a few months after trau$\mathrm{ma}$, that is in a period where natural recovery could still have occurred. The lack of an untreated control group carries some weight in this context. We did consider including a waiting list control but were concerned about the ethical implications of denying earlier treatment to randomly selected patients. Also, because our clinic is located in a large city, there was a possibility of patients looking for and receiving treatment elsewhere if they were allocated to a waiting list group. While comorbid diagnoses on DSM-IV axis I were established, we did not include a measure of depression severity, which would have been an important secondary outcome given the high comorbidity between PTSD and depression. With regard to defining exclusion criteria, a clear operationalization of when a patient would not be able or did not wish to undergo treatment within the stated study protocol is lacking, which is a weakness of the study. Our rather strict exclusion criteria can be seen as another limitation, and our results cannot be generalized to patients with histories of childhood trauma and to patients with severe comorbid depression predating the traumatic event or severe personality pathology. Yet, almost half our sample reported a comorbid affective disorder. The other exclusion criteria served to exclude patients who would normally not be treated with a specific PTSD treatment and PTSD symptoms as well as general psychopathology were not lower than in other studies. Also, criteria only led to the exclusion of 15 patients out of 181 screened. The amount of training which therapists had received in the two study arms cannot be compared directly and might have been different.

For reasons that cannot be explained, there was much more attrition at the posttest time point from the DET condition than the CPT condition, and somewhat (but not statistically significantly) more at the follow-up. As a result, the outcome for a large subgroup of patients who received DET (13 at the posttest assessment; 26 at the follow-up) could not be ascertained. These drawbacks severely limit the inferences that can be drawn from our results.

Although CPT showed greater evidence than DET of improvement on measures of PTSD and related symptoms and cognitions, DET showed promising evidence of generally large effect size improvements using the conservative method of last outcome carried forward and comparable benefits to CPT among study completers. Therefore, further research on DET, including samples exhibiting more comorbidity, is warranted.

\section{References}

1 Bisson J, Andrew M: Psychological treatment of post-traumatic stress disorder (PTSD). Cochrane Database Syst Rev 2009;1:1-48.

2 Cloitre M: Effective psychotherapies for posttraumatic stress disorder: a review and critique. CNS Spectr 2009;14:32-43.
- 3 Bisson JI, Ehlers A, Matthews R, Pilling S, Richards D, Turner S: Psychological treatments for chronic post-traumatic stress disorder. Systematic review and meta-analysis. $\mathrm{Br}$ J Psychiatry 2007;190:97-104. 
4 Bradley R, Greene J, Russ E, Dutra L, Westen D: A multidimensional meta-analysis of psychotherapy for PTSD. Am J Psychiatry 2005; 162:214-227.

5 Seidler GH, Wagner FE: Comparing the efficacy of EMDR and trauma-focused cognitivebehavioral therapy in the treatment of PTSD: a meta-analytic study. Psychol Med 2006;36: 1515-1522.

6 Watts BV, Schnurr PP, Mayo L, Young-Xu Y, Weeks WB, Friedman MJ: Meta-analysis of the efficacy of treatments for posttraumatic stress disorder. J Clin Psychiatry 2013; 74:e541-e550.

7 National Institute for Health and Care Excellence: Posttraumatic Stress Disorder. The Management of PTSD in Adults and Children in Primary and Secondary Care. CG26. London, NICE, 2005.

8 Centre for Posttraumatic Mental Health: Australian Guidelines for the Treatment of Adults with Acute Stress Disorder and Posttraumatic Stress Disorder. Melbourne, ACPMH, 2007.

9 American Psychiatric Association: Practice Guideline for the Treatment of Patients with Acute Stress Disorder and Posttraumatic Stress Disorder. Washington, APA, 2010.

10 Schnyder U: Why new psychotherapies for posttraumatic stress disorder? Psychother Psychosom 2005;74:199-201.

11 Van Emmerik AAP, Kamphuis JH, Emmelkamp PMG: Treating acute and posttraumatic stress disorder with cognitive behavioral therapy or structured writing therapy: a randomized controlled trial. Psychother Psychosom 2008;77:93-100.

12 Kersting A, Dölemeyer R, Steinig J, Walter F, Kroker K, Baust K, Wagner B: Brief internetbased intervention reduces posttraumatic stress and prolonged grief in parents after the loss of a child during pregnancy: a randomized controlled trial. Psychother Psychosom 2013;82:372-381.

13 Jung K, Steil R: A randomized controlled trial on cognitive restructuring and imagery modification to reduce the feeling of being contaminated in adult survivors of childhood sexual abuse suffering from posttraumatic stress disorder. Psychother Psychosom 2013; 82:213-220.

14 Nickerson A, Schnyder U, Bryant RA, Schick M, Mueller J, Morina N: Moral injury in traumatized refugees. Psychother Psychosom 2015;84:122-123.

15 Jacob N, Neuner F, Maedl A, Schaal S, Elbert T: Dissemination of psychotherapy for trauma spectrum disorders in postconflict settings: a randomized controlled trial in Rwanda. Psychother Psychosom 2014;83:354-363.

-16 Paivio SC, Greenberg LS: Resolving 'unfinished business': efficacy of experiential therapy using empty-chair dialogue. J Consult Clin Psychol 1995;63:419-425.
17 Paivio SC, Nieuwenhuis JA: Efficacy of emotion focused therapy for adult survivors of child abuse: a preliminary study. J Trauma Stress 2001;14:115-133.

18 Rosner R, Henkel C: Die Gestalttherapie in der Psychotraumatologie - Charakteristika und Wirksamkeit gestalttherapeutischer Interventionen bei posttraumatischen Belastungsstörungen (Gestalt therapy in psychotraumatology - characteristics and efficacy of gestalt interventions for posttraumatic stress disorder). Trauma Gewalt 2010;4:294-303.

19 Imel ZE, Laska K, Jakupcak M, Simpson TL: Meta-analysis of dropout in treatments for posttraumatic stress disorder. J Consult Clin Psychol 2013;81:394-404.

20 Yontef GM: Awareness, Dialogue and Process: Essays on Gestalt Therapy. Gouldsboro, Gestalt Journal Press, 1993.

21 Butollo W, Hagl M, Krüsmann M: Kreativität und Destruktion posttraumatischer Bewältigung (Creativity and Destruction in Posttraumatic Coping). Munich, Pfeiffer, 1999.

22 Butollo W, Karl R, König J, Hagl M: Dialogical exposure in a gestalt-based treatment for posttraumatic stress disorder. Gestalt Review 2014;18:112-129.

23 Hagl M, Powell S, Rosner R, Butollo W: Dialogical exposure with traumatically bereaved Bosnian women: findings from a controlled trial. Clin Psychol Psychother DOI: 10.1002/ cpp. 1921.

24 Sloan DM, Feinstein BA, Gallagher MW, Beck J, Keane TM: Efficacy of group treatment for posttraumatic stress disorder symptoms: a meta-analysis. Psychol Trauma Theory Res Pract Policy 2013;5:175-183.

25 Butollo W, König J, Karl R, Henkel C, Rosner $\mathrm{R}$ : Feasibility and outcome of dialogical exposure therapy for posttraumatic stress disorder. Psychother Res 2014;24:514-521.

26 Maercker A, Schützwohl M: Erfassung von psychischen Belastungsfolgen: die Impact of Event Skala - revidierte Version (IES-R) [Measurement of psychological sequelae of stress: the Impact of Event Scale - Revised (IES-R)]. Diagnostica 1998;44:130-141.

27 Foa EB, Cashman L, Jaycox L, Perry K: The validation of a self-report measure of posttraumatic stress disorder: the Posttraumatic Diagnostic Scale. Psychol Assess 1997;9:446451.

28 Resick PA, Monson CM, Chard KM: Cognitive Processing Therapy: Veteran/Military Version. Washington, Department of Veterans' Affairs, 2007.

29 Faul F, Erdfelder E, Lang AG, Buchner A: G*Power 3: a flexible statistical power analysis program for the social, behavioral, and biomedical sciences. Behav Res Methods 2007; 39:175-191.

30 American Psychiatric Association: Diagnostic and Statistical Manual of Mental Disorders, ed 4. Washington, APA, 1994.
31 Hiller W, Zaudig M, Mombour W: IDCL Internationale Diagnosen Checklisten für DSM-IV und ICD-10 (IDCL International Diagnostic Checklists for DSM-IV and ICD10). Göttingen, Hogrefe, 1996.

32 Wittchen HU, Pfister H: Diagnostisches Expertensystem für psychische Störungen (DIA-X) [Diagnostic System for Psychiatric Disorders for Experts (DIA-X)]. Frankfurt, Swets \& Zeitlinger, 1997.

33 Wittchen HU, Lachner G, Wunderlich U. Pfister H: Test-retest reliability of the computerized DSM-IV version of the MunichComposite International Diagnostic Interview (M-CIDI). Soc Psychiatry Psychiatr Epidemiol 1998;33:568-578.

34 Strauss B, Schumacher J: Klinische Interviews und Ratingskalen (Clinical Interviews and Rating Scales). Göttingen, Hogrefe, 2005.

35 Wittchen HU, Zaudig M, Fydrich T: Strukturiertes klinisches Interview für DSM-IV (SKID) [Structured Clinical Interview for DSM-IV (SCID)]. Göttingen, Hogrefe, 1997.

36 Weiss D, Marmar C: The Impact of Event Scale-Revised; in Wilson J, Keane T (eds): Assessing Psychological Trauma and PTSD. New York, Guilford, 1997, pp 168-189.

37 Griesel D, Wessa M, Flor H: Psychometric qualities of the German version of the Posttraumatic Diagnostic Scale (PTDS). Psychol Assess 2006;18:262-268.

38 Ehring T, Kleim B, Clark DM, Foa EB, Ehlers A: Screening for posttraumatic stress disorder. What combination of symptoms predicts best? J Nerv Ment Dis 2007;195:1004-1012.

-39 Derogatis LR, Melisaratos N: The Brief Symptom Inventory: an introductory report. Psychol Med 1983;13:595-605.

40 Franke GH: Brief Symptom Inventory von L.R. Derogatis (Kurzform der SCL-90-R) deutsche Version [Brief Symptom Inventory by L.R. Derogatis (short version of the SCL90-R) - German Version]. Göttingen, Beltz Test GmbH, 2002.

41 Geisheim C, Hahlweg K, Fiegenbaum W, Frank M, Schröder B, von Witzleben I: Das Brief Symptom Inventory (BSI) als Instrument zur Qualitätssicherung in der Psychotherapie [The Brief Symptom Inventory (BSI) as an instrument of quality management in psychotherapy]. Diagnostica 2002;48:28-36.

$\checkmark 42$ Foa EB, Ehlers A, Clark DM, Tolin DF, Orsillo SM: The Posttraumatic Cognitions Inventory (PTCI): development and validation. Psychol Assess1999;11:303-314.

43 Horowitz L, Strauss B, Kordy H: Inventar zur Erfassung interpersonaler Probleme deutsche Version (IIP-D) [Inventory of Interpersonal Problems - German Version (IIPD)]. Weinheim, Beltz Test GmbH, 1994.

44 Horowitz LM, Rosenberg SE, Baer BA, Ureño G, Villaseñor VS: Inventory of Interpersonal Problems: psychometric properties and clinical applications. J Consult Clin Psychol 1988; 56:885-892.
Dialogical Exposure Therapy versus

Cognitive Processing Therapy for PTSD
Psychother Psychosom 2016;85:16-26 
45 Schmitt M, Maes J, Schmal A: Gerechtigkeit als innerdeutsches Problem: Auswahl von Indikatoren seelischer Gesundheit (Justice as a problem in unified Germany: selection of indicators of mental health). Fachbereich I Psychologie, Universität Trier, 1995. http:// www.gerechtigkeitsforschung.de/berichte/ beri080.pdf (accessed August 13, 2013).

46 Fahrenberg J, Myrtek M, Wilk D, Kreutel K: Multimodale Erfassung der Lebenszufriedenheit: eine Untersuchung an Koronarkranken (Multimodal measurement of life satisfaction: a study of patients with coronary disease). Psychother Psychosom Med Psychol 1986;36: 347-354.

47 Butollo W, Karl R: Dialogische Traumatherapie. Ein Manual zur Behandlung der posttraumatischen Belastungsstörung (Dialogical Exposure Therapy. A Treatment Manual for Posttraumatic Stress Disorder). Stuttgart, Klett-Cotta, 2012

48 König J, Resick PA, Karl R, Rosner R: Posttraumatische Belastungsstörung. Ein Manual zur Cognitive Processing Therapy (Posttraumatic Stress Disorder. A Manual for Cognitive Processing Therapy). Göttingen, Hogrefe, 2012.
49 Plouffe KA: Paraprofessional intervention utilizing a cognitive processing therapy protocol. Clin Case Stud 2007;6:348-361.

50 Klauer JK: Handbuch kognitives Training (Handbook Cognitive Training). Göttingen, Hogrefe, 2001.

51 Lenhart A: Mittelwertsunterschiede zweier verschieden grosser Gruppen im Prä-PostDesign $\left(d_{k o r r}\right.$ nach Klauer, 2001) [Mean differences between two groups of different sizes in a pre-post design ( $\mathrm{d}_{\text {korr }}$ according to Klauer, 2001)]. http://www.psychometrica.de/effektstaerke.html (accessed April 3, 2015).

52 Brähler E, Horowitz LM, Kordy H, Schumacher J, Strauss B: Zur Validierung des Inventars zur Erfassung Interpersonaler Probleme (IIP) - Ergebnisse einer Repräsentativbefragung in Ost- und Westdeutschland [Validation of the Inventory of Interpersonal Problems (IIP) - results of a representative survey in Eastern and Western Germany]. Psychother Psychosom Med Psychol 1999;49: 422-431.

53 Cohen J: A power primer. Psychol Bull 1992; 112:155-159.

54 Jacobson NS, Truax P: Clinical significance: a statistical approach to defining meaningful change in psychotherapy research. J Consult Clin Psychol 1991;59:12-19.
55 König J, Karl R, Rosner R, Butollo W: Sudden gains in two therapies for posttraumatic stress disorder. Behav Res Ther 2014;60:15-22.

56 Rizvi SL, Vogt DS, Resick PA: Cognitive and affective predictors of treatment outcome in cognitive processing therapy and prolonged exposure for posttraumatic stress disorder. Behav Res Ther 2009;47:737-743.

57 König J, Onnen M, Karl R, Rosner R, Butollo $\mathrm{W}$ : Interpersonal subtypes and therapy response in patients treated for posttraumatic stress disorder. Clin Psychol Psychother 2015, Epub ahead of print.

58 Resick PA, Nishith P, Weaver TL, Astin MC, Feuer CA: A comparison of cognitive-processing therapy with prolonged exposure and a waiting condition for the treatment of chronic posttraumatic stress disorder in female rape victims. J Consult Clin Psychol 2002;70:867-879.

59 Resick PA, Galovski TE, Uhlmansiek MO, Scher CD, Clum GA, Young-Xu Y: A randomized clinical trial to dismantle components of cognitive processing therapy for posttraumatic stress disorder in female victims of interpersonal violence. J Consult Clin Psychol 2008;76:243-258. 